\title{
Molecular Psychiatry in 2003: editorial decisions and feature articles
}

\author{
Molecular Psychiatry (2003) 8, 123-124. doi:10.1038/ \\ sj.mp.4001317
}

Molecular Psychiatry is now a monthly journal. We hope that by publishing 12 issues a year we will meet the needs of our readers and authors, who are responsible for our success. I am often asked of what made this journal so successful. A key element has been the fact that we are an independent publication, which can rapidly respond to new developments in the field. We look at the quality of what is submitted irrespective of geographical, personal, or institutional factors and quality is the basis of editorial decisions. A complex situation has developed as the journal has become the flagship publication in the field. Even as a monthly, with increased pagination, it is possible for us to publish only a small proportion of what we receive. Moreover, we cannot even send all submissions that are received to peer review, as that would completely overwhelm the review system. As we can only publish a fraction of what we receive, it is reasonable to submit only the most competitive material to external peer review.

Our two-tier peer-review system functions as follows: submitted papers are reviewed in the editorial office, with the assistance of board members. If the material is not thought to be competitive for publication, the authors receive via e-mail a letter informing them of that editorial decision. Having processed over 1500 papers since our launch in 1996, we have a very good concept of what is publishable and what cannot successfully compete in the peerreview system. These early rejections can be very disappointing to authors; however, they are ultimately helpful as they facilitate the development of alternate plans in a timely manner.

The second step in the process consists of outside peer-review. In this case, papers are sent to 2-4 experts, some of whom may be in the authors' recommended list. The outcome of this process has become increasingly frustrating for authors as competition for our space increases. For this reason, I will hereby detail the editorial decision-making process. While quality is paramount, and a prerequisite for publication, other additional factors are taken into consideration. Those include items such as novelty, general interest, topic, number of other accepted articles on the topic, and balance. A highly rigorous study that is the fifth paper with the same conclusion may be superseded by something else that is perhaps not as complete, but that shows something that is new and exciting. Authors do not know what is in press, so it can be frustrating to see one's material rejected without documentation of what else is in production.

The content of the peer-review comments is also a source of questions to us. First, authors must be informed of the following: reviewers send us two forms on each paper. One of those is confidential to me, the other is to be shared with others. Often the most negative comments are stated in the confidential part, and for that reason authors cannot at times understand why their papers were rejected. An additional source of frustration is the following. Given the stiff competition for our space, papers are accepted based on positive, enthusiastic reviews. We need a strong justification for publication. The lack of negative comments in lukewarm reviews is not a mandate for publication. On the contrary, lukewarm reviews that do not reflect a high level of enthusiasm on the reviewer's part are a signal for us not to publish a paper.

An additional factor that negatively impacts on the review process is the statement (usually in the confidential comments to me) that the paper in question was reviewed by the same referee for another journal, rejected there, and then submitted to us without modification. The peer-review system functions in such a way that as a paper bounces from journal to journal it frequently ends up in the same reviewer's desk (or computer screen). It is very frustrating for the referee and the editor to find out that comments had been previously made on the work, that such comments were totally ignored, and that the authors simply repackaged the article, without concern for the referees' critiques, and sent it unchanged to Molecular Psychiatry. This is not acceptable to us. On the other hand, referees are usually favorably impressed when a paper that is rejected by one journal is revised in a manner that adequately addresses their comments, prior to submission to another journal. Such successful revision often requires the performance of additional experiments. Authors may feel pressured to publish fast without doing any additional work, but such efforts often backfire.

Human nature is such that to compete for grants, promotions, and bright trainees one needs distinctive accomplishments. In order for publication in a specific journal to be of value to one's career, it is necessary to make it difficult to publish there. In other words, if everyone published whatever observations they made in Molecular Psychiatry, there would be no reason for us to exist. We have become a competitive 
repository of the best data in our field. Consequently, we cannot offer publication to all authors who contact us. On the contrary, the nature of our business is such that in order for some to succeed, it is necessary that others fail.

I fully understand the frustration of those who are denied publication. As a working scientist I intermittently experience that same type of frustration myself, and I can therefore fully empathize with authors whose work is rejected. However, there is not much that can be done to remedy this. We cannot publish all material that is received. We cannot even publish all highly meritorious material that is received.

It would be more straightforward to require objective criteria for publication. Those might include minimum number of SNPs or haplotypes, $n$, and effect size. However, in actuality, a small effect of a novel gene can be highly interesting, while papers that meet any criteria that we could develop might still be of limited interest to our readers. We therefore prefer to look at each article individually.

There is also the crucial issue of balance. We cannot publish papers that fit a formula. What makes the journal interesting and successful is the fact that we are the premier publication on translational studies in psychiatry. In order to reach that position and maintain it, it is necessary that we publish side by side large studies and smaller observations, research done exclusively in the bench alongside animal work and patient-oriented investigation. Those projects use a variety of methods, from the clinical to the molecular, including work from disciplines as diverse as molecular biology, genetics, neuroscience, imaging, pharmacology, physiology, pathology, and clinical medicine. We can therefore only publish a handful of articles from any one topic, area, and method.

Genetic association studies represent from the editorial perspective a particularly difficult type of submission. Papers that look at one SNP in one disease can only be published if there is something extremely compelling in the data. In this day and age, there is no reason for not looking at all SNPS in a gene or in a biological system and for that reason piecemeal work that is submitted to us will no longer be accepted unless there is a really strong rationale for consideration of the data. Data on the biological relevance of reported allelic variants greatly strengthen submitted articles. As failure to replicate is a major issue in the field, priority will be given to papers that include data from at least two samples. Analysis of haplotypes will be increasingly required for publication.
As in any other type of human endeavor there can be error. A not so relevant paper may receive inappropriately enthusiastic reviews and be published. Or a highly important submission may be negatively reviewed by reviewers who did not understand the importance of the findings. To ensure fairness to all, we have established a formal appeals process. To participate in the formal appeal process, authors should contact our editorial office and request our newly developed appeal forms. I must emphasize that the appeals which may succeed are only those in which the reviewers made factual errors, or the importance of the findings was not understood. It is not productive to appeal lack of scientific priority, or subjective editorial considerations, such as estimation of limited interest to our readership, inability to compete successfully for space with other recently accepted papers, or editorial necessity to achieve balance among different types of submissions. I hope these comments can help prospective authors as they navigate through our submission and review process.

To end this editorial, I would like to note a new section in Molecular Psychiatry. That section will contain Feature Articles, which are comprehensive, state-of-the-art reviews, written by recognized experts. We expect that those articles will rapidly become key references in our field, and the primary texts in their areas. Our cover paper, by Lenox and Wang, is our first Feature Article. It provides an overview of the molecular biology of lithium (pp. 134-143). We will expedite publication of Feature Articles to ensure that they present the latest and most current information on a topic. While those articles are being commissioned by invitation, we may consider unsolicited submissions to this section. Prospective authors should follow the following procedure: first they should contact the editorial office (molecularpsychiatry@mednet.ucla.edu), indicating the topic of the proposed article and documenting their expertise. If there is no substantial overlap with articles that are in press or in review, and if we feel that the topic would be of interest to a broad readership, we will solicit an outline of the paper, to be followed by a formal submission, which will go out for peer review. We hope that our readers find upcoming Feature Articles an exciting source of current information on hot topics in Molecular Psychiatry.

J Licinio, MD UCLA Laboratory for Pharmacogenomics E-mail: Licinio@ucla.edu 